\title{
A comparative study of impulse-resistant metal sandwich plates
}

\author{
Zhenyu Xue, John W. Hutchinson* \\ Division of Engineering and Applied Sciences, Harvard University, Pierce Hall, Cambridge, MA 02138, USA
}

Received 11 March 2003; received in revised form 19 August 2003; accepted 24 August 2003

\begin{abstract}
The performance of metal sandwich plates under impulsive blast loads is compared to that of solid plates made of the same material and having the same weight. Three core geometries are considered: pyramidal truss, square honeycomb and folded plate. Plates of infinite length and clamped along their sides are subject to uniform impulsive load. The momentum impulse is applied to the face sheet towards the blast in the case of the sandwich plate, while it is distributed uniformly through the thickness of the solid plate. Large impulses are considered that are sufficient to produce lateral plate deflections more than $10 \%$ of the plate width. Fracture is not considered; the plates are assumed to have sufficient ductility to be able to sustain the deformations. A limited study of weight optimization is carried out for each of the core types with respect to the respective geometric parameters, including core and face sheet thickness, core member aspect ratios and relative density. A well-designed sandwich plate can sustain significantly larger blast impulses than a solid plate of the same weight. If the blast medium is water, fluid-structure interaction can reduce the momentum imparted to a sandwich plate by almost a factor of two relative to that imparted to a solid plate of the same weight, and, consequently, the relative benefit of the sandwich plate is significantly enhanced over its solid counterpart.
\end{abstract}

(C) 2003 Elsevier Ltd. All rights reserved.

Keywords: Sandwich cores; Sandwich plates; Optimization; Finite element method; Impulse load

\section{Introduction}

Blast-resistant structure is important not only in military applications but also in the chemical, oil, and nuclear industries. Most structures designed to withstand large blast loading employ solid-wall plate and shell components. Here, we explore the advantages and disadvantages of

\footnotetext{
*Corresponding author. Tel.: + 1-617-495-2848; fax: + 1-617-495-9837.

E-mail address: hutchinson@husm.harvard.edu (J.W. Hutchinson).
} 


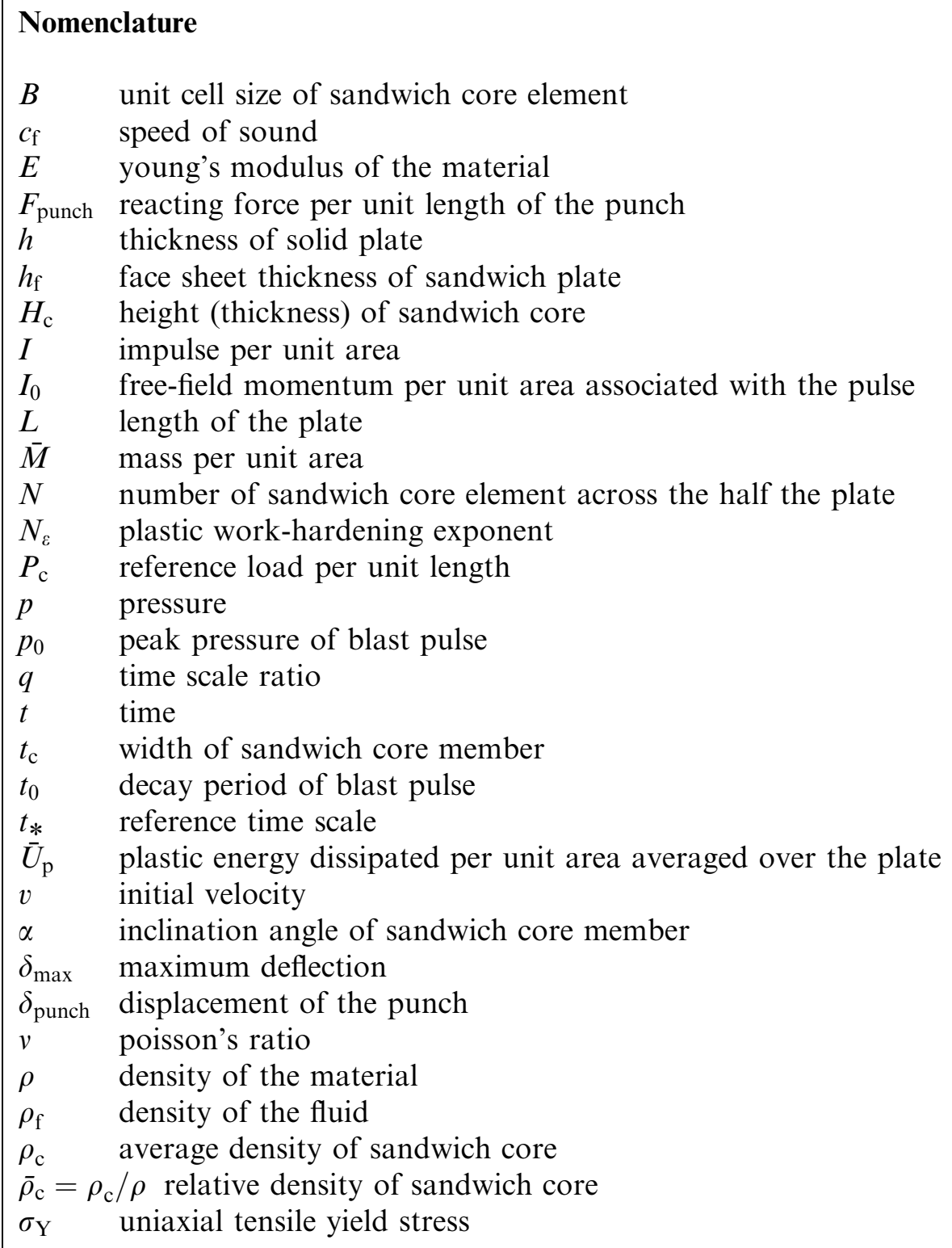

metal sandwich plates relative to solid plates made of the same material and having the same total weight under blast loads. This paper is a sequel to an earlier paper [1] that investigated the relative advantage of clamped circular sandwich plates with a tetragonal truss core over equal weight solid plates subject to identical initial momentum impulses. The finding of the earlier paper was that sandwich plates could indeed outperform solid plates unless exceptionally large deflections could be tolerated and sustained without fracture. That finding was tentative for two reasons. First, a continuum model of the core was used in the study that did not capture details of the dynamic plastic buckling of the truss elements. Second, the study did not account for fluid-structure 
interaction of the blast pulse and the plate. Fluid-structure interaction significantly affects the momentum imparted to the sandwich plate relative to the solid plate of similar weight. While the effect in air is small, for blast pulses transmitted in water, the momentum imparted to a metal sandwich plate is substantially less (by a factor of almost two, typically) than that imparted to the solid plate of equal mass. Fluid-structure interaction provides a significant additional enhancement to the benefit of sandwich plate construction for blast resistance in a water environment.

In this paper, we employ highly refined three-dimensional finite element modeling of the geometry of the sandwich plates to compute their resistance to impulsive blast loads. A first attempt will be made to optimize the plates to achieve maximum performance at a specified weight. In addition, the substantial benefit of sandwich construction over solid plates for water blasts due to fluid-structure interaction will be brought out. The study focuses on the maximum deflections that the plates sustain. Efforts to account for fracture will be undertaken in subsequent work; in this paper, the material is assumed to be sufficiently ductile to withstand the deformations produced by the blast.

\subsection{Three types of sandwich cores}

Infinite plates of width $2 L$ that are clamped along their edges are considered, as depicted schematically in Fig. 1. Three core geometries are analyzed: pyramidal truss, square honeycomb, and folded (or corrugated) plate. Pyramidal truss core sandwich plates have recently been manufactured for relatively low cost applications, such as decking for transport ships. Folded plate core sandwich structure is available commercially under the trade name "Navtruss". Each of the three types of sandwich plate has a basic unit that is repetitive in the long direction (perpendicular to the plane of the cross-section in Fig. 1). The loading is taken to be independent of the coordinate in the long direction, and thus it is possible to analyze just one three-dimensional unit of the plate that repeats periodically along its length. Fig. 2a shows the pyramidal truss core plate, along with the periodic unit used in the finite element mode in Fig. 2b. Similarly, the periodic unit used to analyze the plate with the square honeycomb core is shown in Fig. 3. The

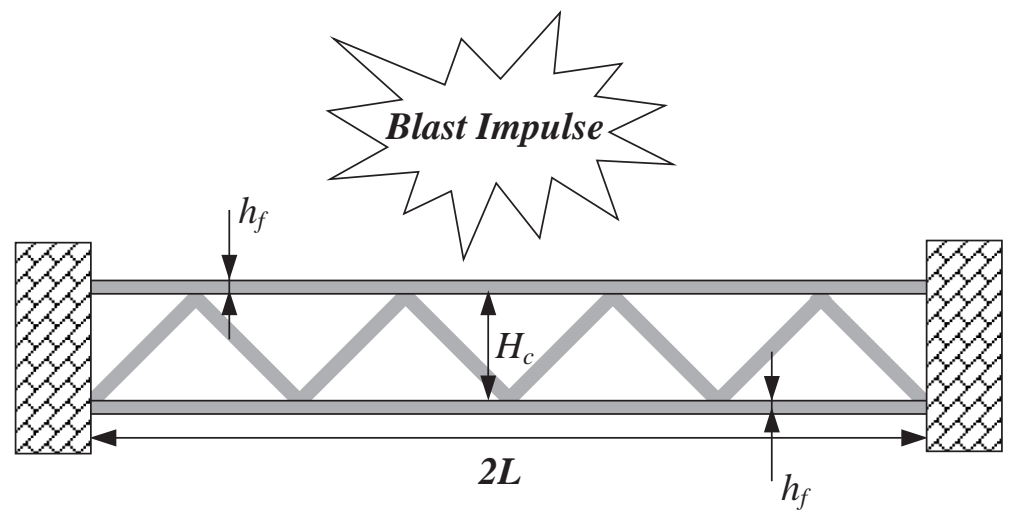

Fig. 1. Schematic diagram of a sandwich structure clamped at both ends under blast loading. 


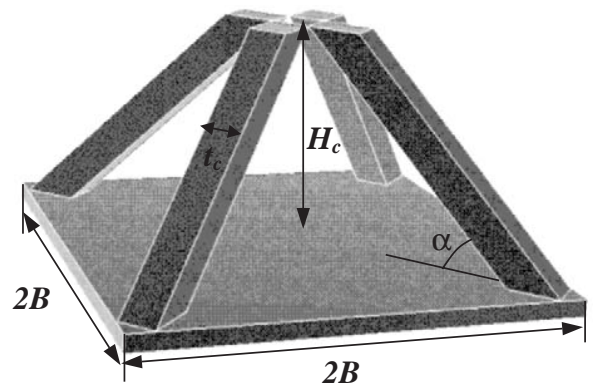

(a)

(b)

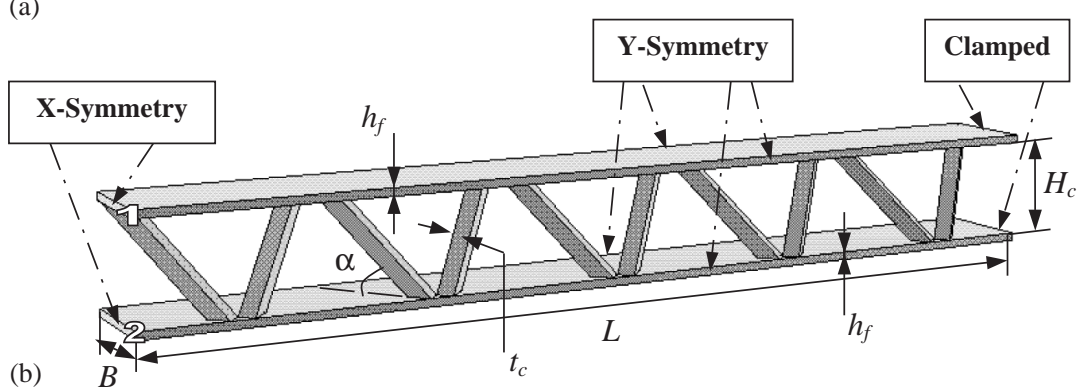

Fig. 2. (a) Schematic diagram of pyramidal truss core configuration. (b) Schematic diagram of the computation model of sandwich panel with pyramidal truss core configuration.

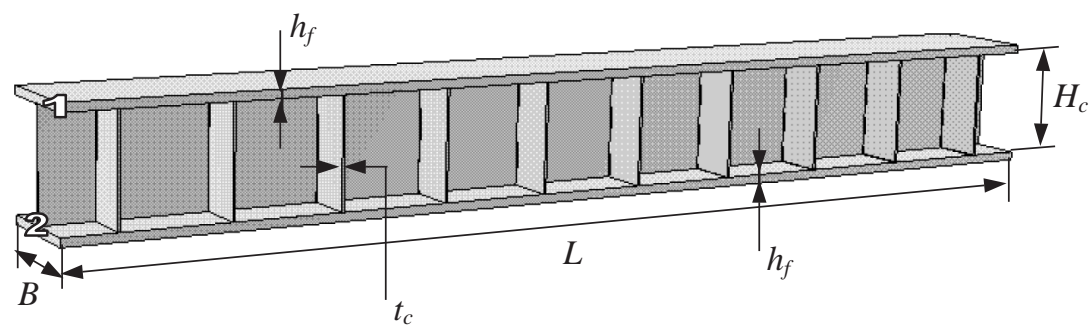

Fig. 3. Schematic diagram of the computation model of sandwich panel with square honeycomb core configuration.

folded plate core sandwich is illustrated in Fig. 4a, and its repeating unit used in the finite element model is shown in Fig. 4b. Note that core webs of the folded plate run across the width of the plate and not parallel to the long direction. For each of the three types of plate structures, all components (face sheets, core plate webs, or truss members) of the basic unit are fully meshed with three-dimensional elements. The solid plate used as the reference is analyzed in the same manner. The commercial code, ABAQUS Explicit, is used to carry out the dynamic calculations while the implicit version is used for the quasi-static computations. In all the calculations, eightnode brick elements are employed with reduced integration. Periodic boundary conditions are applied at each end of the repeating unit in the long direction, and clamped conditions are imposed along the two sides. Calculations in this paper have been performed for plates constructed from a stainless steel whose stress-strain behavior will be specified subsequently. 
(a)
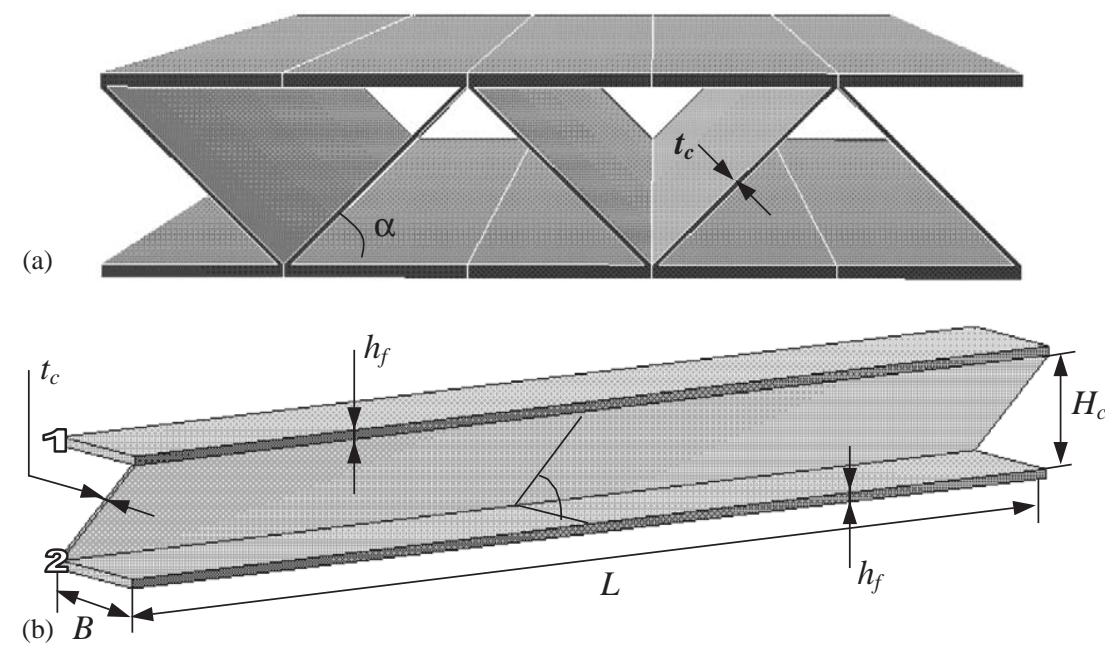

Fig. 4. (a) Schematic diagram of folded plate core configuration. (b) Schematic diagram of the computation model of sandwich panel with folded plate core configuration.

\subsection{Idealized blast loading}

Plate response to one set of quasi-static loads will be presented to illustrate certain aspects of the behavior of sandwich relative to solid plates. However, most of the results presented will be for the dynamic response to impulsive blast-type loads. In all cases, a uniform impulse per unit area, $I$ $\left(\mathrm{Ns} / \mathrm{m}^{2}\right)$, is applied to the plate at time $t=0$. For the solid plate, this impulse is distributed uniformly through the thickness as a uniform initial velocity normal to the plate, $v=I / \rho h$, where $\rho$ is the density of the material and $h$ is the plate thickness. For the sandwich plates, the impulse is applied only to the face sheet towards the blast, again as a uniform initial velocity, $v=I / \rho h_{\mathrm{f}}$, where $h_{\mathrm{f}}$ is the face sheet thickness. The rationale for replacing the pressure pulse acting on the plate by an initial impulse rests on the fact that the period of the blast pulse is short compared to the response time of the plate [2]. The period, $t_{0}$, characterizing the leading portion of a blast pulse, is typically on the order of a tenth of a millisecond whether in air or water. The response time for steel plate structures of the type considered here with width of the order of a meter or more is measured in several milliseconds. Structural response times will be highlighted in the paper, as will the limitations of the approach based on imposition of an initial impulse.

\subsection{Fluid-structure interaction}

Early work by Taylor [3,4] used the solution for a one-dimensional wave pulse impinging on a solid to compute the momentum transmitted to the plate by the blast pulse. The backside of the plate is unrestrained. Let $\rho_{\mathrm{f}}$ be the density of the fluid on the side of impinging pulse and $c_{\mathrm{f}}$ its speed of sound. Let $\rho$ be the density of the plate and $h$ its thickness. Following Taylor and also more recent work on representative blast pulses [5], take the time variation of the free-field pressure at any point in the fluid engulfed by the pulse starting at $t=0$ to be $p=p_{0} \mathrm{e}^{-t / t_{0}}$ for $t>0$, where $p_{0}$ is the pulse peak and $t_{0}$ is its decay period. The free-field momentum 
per unit area associated with the pulse is $I_{0}=\int_{0}^{\infty} p \mathrm{~d} t=p_{0} t_{0}$. When this pressure pulse impinges on the plate it sets the plate in motion and is partly reflected. The time at which the plate achieves its maximum velocity coincides with onset of tensile stress (negative pressure) at the interface between the fluid and the plate, leading to cavitation in the fluid shortly thereafter. Basing the momentum per unit area, $I$, imparted to the plate on the maximum velocity, one obtains Taylor's relation

$$
\frac{I}{I_{0}}=2 q^{q /(1-q)}
$$

where $q=t_{0} / t_{*}$. The time scale in this relation characterizing the fluid-plate interaction is

$$
t_{*}=\frac{\rho h}{\rho_{\mathrm{f}} c_{\mathrm{f}}} .
$$

The momentum ratio, $I / I_{0}$, is plotted as a function of the time scale ratio, $q=t_{0} / t_{*}$, in Fig. 5 . The two limits have simple interpretations. If $t_{0} / t_{*} \ll 1$, the plate is relatively massive, hardly moving, and the pulse is reflected with nearly perfectly "bounce" such that the reflected free-field momentum is nearly $-I_{0}$. The plate acquires momentum approaching $2 I_{0}$. In the limit where $t_{0} / t_{*} \gg 1$, the plate is thin having little effect on the fluid motion. Approximately, the plate acquires the velocity of the fluid computed as if the wave impinged on a free surface. In this limit, one can readily show that $I / I_{0} \cong 2 t_{*} / t_{0}$, in agreement with Eq. (2).

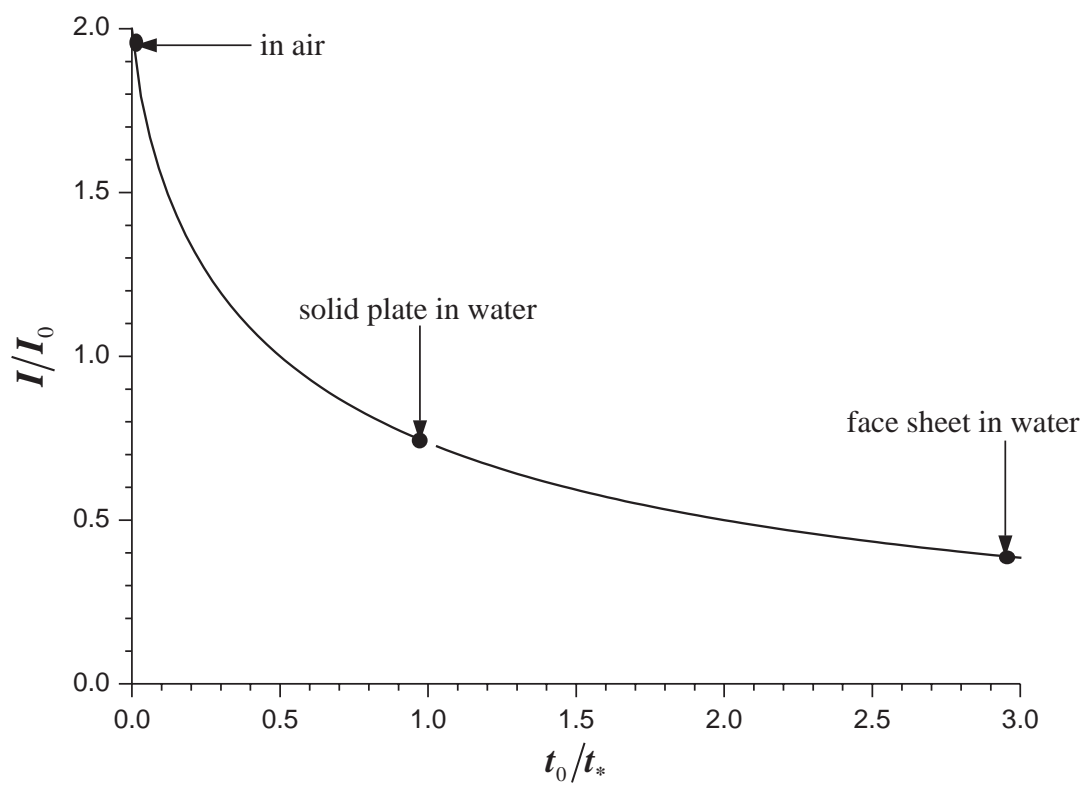

Fig. 5. Taylor's [2, 3] plot for assessing fluid-structure interaction. $I_{0}$ is the free-field momentum per area of the incident blast pulse, $I$ is the momentum per area imparted to the plate, $t_{0}$ is the pulse period, and $t_{*}$ is a time scale characterizing the fluid-structure interaction defined in Eq. (2). 
The importance of fluid-structure interaction is revealed by considering representative time scales for a steel plate with thickness $0.0254 \mathrm{~m}$ in air and in water:

$$
\begin{aligned}
& t_{0} \approx 10^{-4} \mathrm{~s} \text { (water or air), } \\
& t_{*} \approx 1.02 \times 10^{-4} \mathrm{~s} \text { (water), } \\
& t_{*} \approx 0.39 \mathrm{~s} \text { (air) }
\end{aligned}
$$

From Fig. 5, it is evident that the plate in air $\left(q=t_{0} / t_{*} \approx 2.5 \times 10^{-4}\right)$ is so small that the maximum possible momentum, $2 I_{0}$, is transferred to the plate. (Taylor's result (1) requires modification for air blasts to account for nonlinear effects and the presence of air on the backside of the plate, but the conclusion concerning momentum transfer to the plate holds.) On the other hand, in water, the time scales of the pulse and the fluid-structural interaction are of the same order for the plate $\left(q=t_{0} / t_{*}=0.98\right)$ and the momentum transferred to the plate by the blast pulse is less than one half the maximum possible (cf. Fig. 5).

This same plot can be used to illustrate the potential benefit of a sandwich plate from the vantage point of designing for reduction of momentum transfer to the structure. It will be seen later in the paper that the mass per area of an optimally designed sandwich plate is distributed with roughly one third to each of the face sheets and one third to the core. If the sandwich plate is compared to a solid plate of the same material and total mass, the thickness of face sheet of the sandwich exposed to the initial blast impulse will be roughly one-third that of the solid plate. Thus, rather than $t_{*}=1.02 \times 10^{-4} \mathrm{~s}$, which holds for the solid plate in water, the face of the sandwich plate has $t_{*}=0.34 \times 10^{-4} \mathrm{~s}$. The result can be seen in Fig. 5: the momentum transferred to the sandwich face is reduced to about one half that transferred to the solid plate. This momentum reduction is an enormous benefit for sandwich plates in water (but not in air), as will be seen in more detail in the last section of the paper. We will also provide the argument underlying neglect of the interaction of the face sheet towards the blast with the rest of the sandwich plate during the period when the pressure pulse strikes.

\subsection{Outline of the paper}

Details of the computational models for sandwich structures with the three types of core are presented in Section 2. The material is taken to be a stainless steel (\#304) that has relatively high work hardening. Material strain rate sensitivity is not accounted for in the present study, nor is fracture. The material is assumed to be sufficiently ductile to sustain the stresses and strains experienced by the plate at the maximum deflection. Parametric equations for each structure describe the relation among all its geometric parameters.

In Section 3, the force-displacement response of sandwich plates with the three types of core subjected to a quasi-static indentation load is investigated using ABAQUS standard [6]. The responses are compared with that of a solid plate made from the same material and having the same mass. The quasi-static study highlights the underlying mechanics of bending and stretching of sandwich structure under large deformation and the several mechanisms for energy dissipation.

Studies aimed at achieving the near-optimization of the sandwich plates with the three basic core configurations subjected to blast load are performed in Section 4. These computations are preformed using ABAQUS explicit [7]. For a given face-sheet/core assembly with specified mass 
per unit area and subject to a prescribed momentum impulse per area, optimization is based on minimizing the maximum permanent deflection of the bottom face sheet. While full optimization is not achieved, considerable insight is obtained into sandwich plate design for effective blast resistance. For a given impulse level, the maximum deflection of each sandwich structure is compared to that of the corresponding solid plate having same mass in order to evaluate its relative efficiency. While applicable to air blasts, these comparisons do not account for the benefit enjoyed by sandwich plates of the reduction in the momentum impulse in water due to fluidstructure interaction. Nevertheless, as anticipated in the earlier study [1], there is a significant impulse range over which a well-designed sandwich plate can outperform its solid counterpart. The influence of fluid-structure interaction on the relative performance of sandwich plates in a water environment is accounted for in Section 5, where it will be seen that the potential of sandwich plates as blast-resistant structures is indeed substantial.

\section{Specification of sandwich plates and finite element modeling}

In this section each of the three types of sandwich plates introduced in Section 1.1 will be specified in detail with discussion of aspects of the finite element modeling. The plates are fully clamped along their sides, corresponding to face sheets welded to rigid walls. All components of the plate are made from Stainless Steel (\#304) with density, $\rho=8000 \mathrm{~kg} / \mathrm{m}^{3}$. A piecewise function has been fit to the elastic-plastic tensile behavior of the material giving

$$
\sigma= \begin{cases}E \varepsilon, & \varepsilon \leqslant 0.002 \\ \sigma_{\mathrm{Y}}(\varepsilon / 0.002)^{N_{\varepsilon}}, & \varepsilon>0.002\end{cases}
$$

with Young's modulus $E=102.5 \mathrm{GPa}$; Poisson's ratio $v=0.3$; tensile yield stress $\sigma_{\mathrm{Y}}=205 \mathrm{MPa}$, and the plastic strain hardening exponent $N_{\varepsilon}=0.17$ (all values are taken from Metals handbook [8]). Isotropic hardening flow theory is employed based on the Mises yield criterion. The results will be presented in non-dimensional that reveal how the structural designs scale with $\sigma_{\mathrm{Y}}$. However, no attempt has been made to vary the work hardening exponent, $N_{\varepsilon}$. Although this is not a dominant material parameter, it does influence the performance of the sandwich plate.

As mentioned earlier, three types of cores have been considered: pyramidal truss, square honeycomb, and folded plate. The pyramidal truss core will be used to illustrate the finite element modeling with brief comments on aspects unique to the other two.

\subsection{Pyramidal truss core}

A pyramidal truss core element is configured in Fig. 2a. All members of the core are identical with height $H_{\mathrm{c}}$ and a solid square cross-section of width $t_{\mathrm{c}}$. At the face sheet, each truss member has three neighbors coming into contact at their corners with angle of inclination between truss and face sheet, $\alpha$. The geometry at the top and bottom faces is also identical. Each pyramidal unit has a bottom area of $2 B \times 2 B$ where

$$
B=\left(t_{\mathrm{c}}+t_{\mathrm{c}} / \sin \alpha+H_{\mathrm{c}} / \tan \alpha\right) / \sqrt{2} .
$$


The half-width $L$ is given by $L=N B$, where $N$ is the number of truss members across the half the plate. The relative density of this core, $\bar{\rho}_{\mathrm{c}}$, is defined as the ratio of average density of the core, $\rho_{\mathrm{c}}$, to the density of the material, $\rho$, or, equivalently, as the volume fraction of the core occupied by material. It can be expressed in terms of $H_{\mathrm{c}}, t_{\mathrm{c}}$, and $\alpha$ as

$$
\bar{\rho}_{\mathrm{c}}=\frac{\rho_{\mathrm{c}}}{\rho}=\frac{2 t_{\mathrm{c}}^{2}}{\left(t_{\mathrm{c}}+t_{\mathrm{c}} / \sin \alpha+H_{\mathrm{c}} / \tan \alpha\right)^{2} \sin \alpha} .
$$

For all three plate types, the normalized mass per unit area of the plate, $\bar{M} / \rho L$, is given by

$$
\frac{\bar{M}}{\rho L}=\left(2 \frac{h_{\mathrm{f}}}{L}+\bar{\rho}_{\mathrm{c}} \frac{H_{\mathrm{c}}}{L}\right) .
$$

If $\bar{M}$ and $L$ are specified, the geometry of the pyramidal truss core sandwich plate is specified by three variables. In the optimization study, $H_{\mathrm{c}} / L, \bar{\rho}_{\mathrm{c}}$ and $\alpha$ will be chosen as the parameters to be varied. Note that the face sheet thickness, $h_{\mathrm{f}} / L$, is then determined in terms of $\bar{\rho}_{\mathrm{c}}$ and $H_{\mathrm{c}} / L$ by Eq. (7). Calculations were necessarily carried out with an integral number of truss members, $N$. Thus, variations in the core cell size $B$ leads to changes in $L$. In the optimization studies, $N$ was chosen as that integer maintaining $L$ as close to the specified value as possible. Since $N$ is fairly large the discrepancy from the specified value is small.

Symmetry of a unit segment of the sandwich plate in the lengthwise direction, together with the uniformity of the loading, permits one to analyze the half-unit shown in Fig. 2b. Symmetry boundary conditions are imposed on the surfaces of the face sheets along the plate centerline and on the front and back surfaces of the face sheets in the long direction. Nodal displacements of the face sheets are set to zero along the clamped edge. Additionally, symbols 1 and 2 in Fig. $2 b$ mark the positions of the nodes on top face sheet and bottom face sheets, respectively, where the maximum deflections occur and which will be reported below.

\subsection{Square honeycomb core}

Fig. 3 shows the periodic unit employed in the finite element model of the sandwich plate with the square honeycomb core. The half-width of the sandwich plate is again $L$, the face sheets have thickness, $h_{\mathrm{f}}$, the core height of core is $H_{\mathrm{c}}$, and the wall thickness of the core webs is $t_{\mathrm{c}}$. The unit cell size of core element is $B$. The integer number of unit core elements across the half-width is $N$ such that $L=N B$. The relative density of core is

$$
\bar{\rho}_{\mathrm{c}}=\frac{N\left(B-t_{\mathrm{c}}\right) t_{\mathrm{c}}+t_{\mathrm{c}} L}{B L} .
$$

With $L$ and $\bar{M}$ specified, the three independent parameters determining the geometry can be taken as $\bar{\rho}_{\mathrm{c}}, H / L$ and $B ; h_{\mathrm{f}} / L$ is obtained from Eq. (7) and $t_{\mathrm{c}} / L$ from Eq. (8). The nodes on the top and bottom face sheets where the displacements are reported are indicated in Fig. 3. The boundary conditions are similar to those for pyramidal truss core except that all constrains have been applied not only to the edges of face sheets but also to the corresponding edges of honeycomb core elements. 


\subsection{Folded plate core}

The sandwich plate with folded plate core is depicted in Fig. 4. The sandwich plate has halfwidth $L$, core height $H_{\mathrm{c}}$, core plate thickness $t_{\mathrm{c}}$, face sheet thickness $h_{\mathrm{f}}$, and core inclination angle $\alpha$. The folded plate core is oriented with its folds aligned in the width direction, perpendicular to the edges along which the sandwich plate is clamped. This orientation exploits the stretching capacity of the core when the deflections become sufficiently large. The periodic unit employed in the finite element analysis is displayed in Fig. 4b. The relative density of the core is

$$
\bar{\rho}_{\mathrm{c}}=\frac{t_{\mathrm{c}}}{t_{\mathrm{c}}+H_{\mathrm{c}} \cos \alpha} .
$$

The three independent variables employed in the optimization are: $\bar{\rho}_{\mathrm{c}}, H_{\mathrm{c}} / L$, and $\alpha$. The boundary conditions applied to the edges of the unit in Fig. $4 \mathrm{~b}$ are similar to those discussed for the other two cases.

All simulations of the dynamic behavior of sandwich plates with the three core configurations have been made using ABAQUS/Explicit [7]. Meshes were generated with the help of ABAQUS/ CAE software. As mentioned earlier, eight-node linear brick elements with reduced integration were used to model all components of the sandwich structures to aid consistency of the numerical results. Limited studies with shell elements for modeling the webs of the honeycomb and folded plate cores indicated that those elements would be an acceptable alternative. Additional studies showed that meshing used for all the results reported is adequate to limit the numerical error for the maximum deflection to $1 \%$. Subsequent work on fracture modes of all metal sandwich plates will undoubtedly require finer meshes in regions where failure occurs.

The explicit version of ABAQUS is also able to accurately predict the plastic buckling of the core structure under large deformation. Separate calculations have been made focusing specifically on the fidelity of the analysis on the dynamic plastic buckling of truss and web elements loaded in the manner of element in the sandwich plate under the blast loading. These calculations have demonstrated the ability of the ABAQUS code with the meshed used to accurately model the dynamic response of the elements. Moreover, additional calculations showing trends for systematic variations of geometric or material imperfections in core members (not be reported because of space limitations) indicated that the results reported below are not significantly altered by small geometric imperfections.

\section{Force-displacement response of sandwich structures under quasi-static loads}

A bend-stretch test of the sandwich panel can be set up as in the schematic in Fig. 6 . The three core geometries specified in Section 2 are analyzed. A rigid flat punch of width $2 a$ and infinite in extent in the direction perpendicular to the cross-section shown is applied to the center of the top face sheet. As the displacement of the punch $\delta_{\text {punch }}$ increases, the magnitude of reacting force per unit length $F_{\text {punch }}$ is computed. The force-displacement relation will be compared with that computed for a solid plate made of the same material and having precisely the same width and mass per area. 
(a)
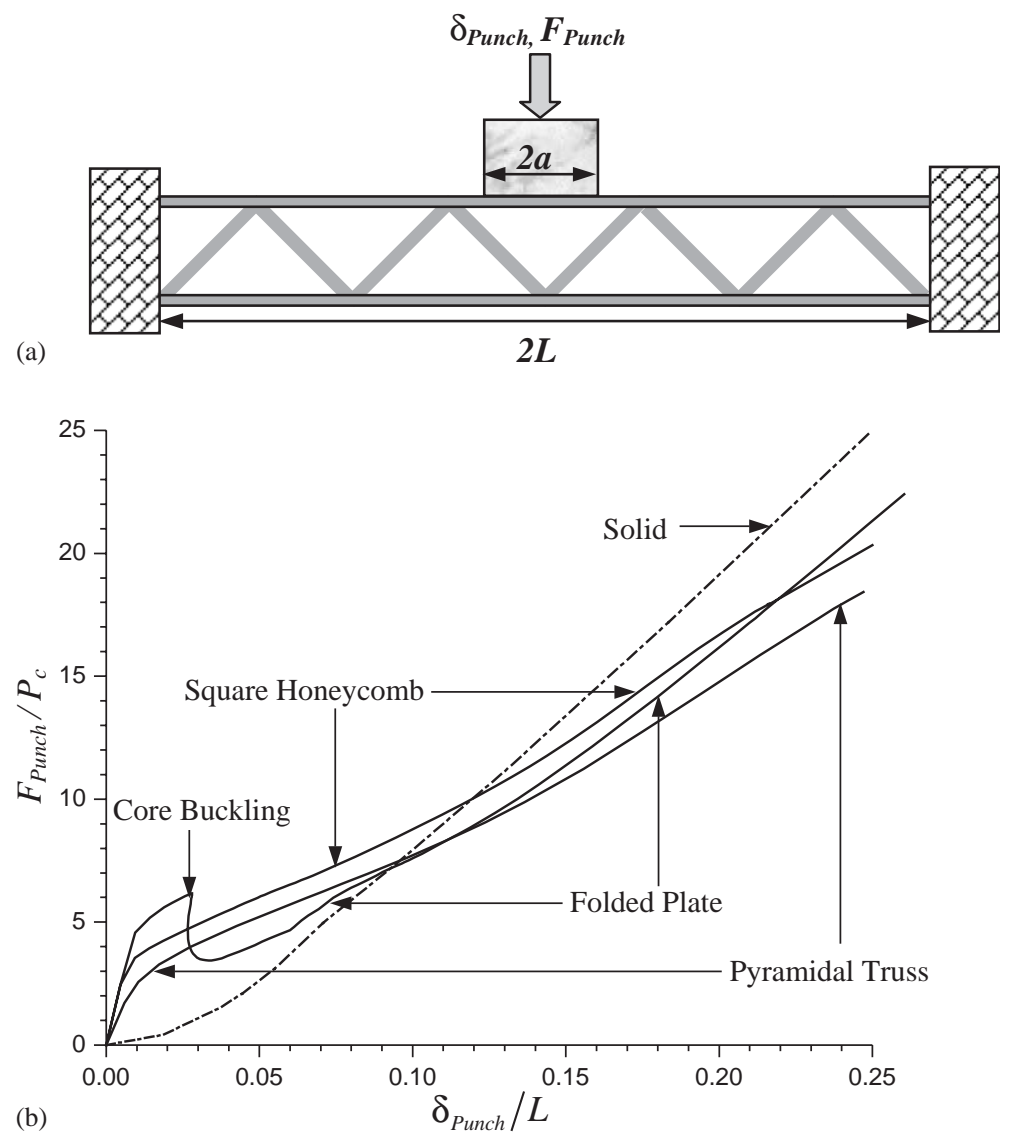

Fig. 6. The quasi-static force-displacement responses of clamped sandwich structures with various core configurations subjected to quasi-static indentation load. The response of the solid plate having the same total mass, $\bar{M} /(\rho L)=0.02$, is also plotted for comparison. The parameters specifying the plates are specified in the text.

The plate geometries and finite element models described in Section 2 are used along with ABAQUS Standard to compute the responses. Eight-node linear brick elements with reduced integration are used to model the structures, while the flat punch is modeled by a rigid element. It is assumed that the contact area between the punch and the plate does not allow tangential slip.

A reference load per unit length, $P_{\mathrm{c}}$, is defined corresponding to the limit load per unit length in bending of a solid, clamped, elastic-perfectly plastic plate of width, $2 L$, having thickness, $h$, yield stress, $\sigma_{\mathrm{Y}}$, which is subject to a concentrated centerline force:

$$
P_{\mathrm{c}}=\sigma_{\mathrm{Y}} h^{2} / L
$$

Here, the value of $\sigma_{\mathrm{Y}}$ is the initial tensile yield stress of the tensile stress-strain curve (4) defined earlier and $h=\bar{M} / \rho$ is taken as thickness of the comparison solid plate having the same mass per area as the sandwich plates. Fig. 6 presents the relation between the normalized punch force, $F_{\text {punch }} / P_{\mathrm{c}}$, and the normalized punch displacement, $\delta_{\text {punch }} / L$, for representative examples of each of the three sandwich structures and for the solid plate of equal mass. In these examples, the three 
sandwich plates each have relative density, $\bar{\rho}_{\mathrm{c}}=0.04$ and normalized core height, $H_{\mathrm{c}} / L=1 / 10$. It follows from Eq. (7) that for all the sandwich plates considered, $h_{\mathrm{f}} / H_{\mathrm{c}}=0.08$, corresponding to core mass of exactly $1 / 5$ the total mass. For all four plates, $\bar{M} / \rho L=0.02$ and $a / L=0.1$. The problems are specified in dimensionless terms, but note that for stainless steel plates with $L=1 \mathrm{~m}$, $\bar{M} / \rho L=0.02$ corresponds to a solid plate with thickness, $h=0.02 \mathrm{~m}$. In addition to these normalized values and the stress-strain relation (4), the truss core plate is completely specified by $\alpha$ which is taken to be $45^{\circ}$ for the plate in Fig. 6 . The square honeycomb plate is specified by the choice $B / H_{\mathrm{c}}=1$ (giving $t_{\mathrm{c}} / H_{\mathrm{c}}=0.022$ ), and the folded plate core sandwich plate in Fig. 6 is fully specified by $\alpha=45^{\circ}$ (giving $t_{\mathrm{c}} / H_{\mathrm{c}}=0.0295$ ). The solid plate has $h / L=0.02$.

Numerical results for the equal mass solid plate are also shown as the dash line in Fig. 6. It is well known that during the beginning stage of deformation of a plate in the bend-stretch experiment, bending dominates the behavior of the system such that there exists in-plane compressive stresses on the top surface of the plate and in-plane tensile stresses on the bottom surface. Then, as the displacement of the punch reaches about one plate thickness, the stress on the top surface gradually changes from compression to tension signaling the transition to stretching. The dimensions of the solid stainless steel plate in Fig. 6 are such that the plate does not yield until stretch dominates bending, which occurs for $\delta_{\text {punch }} / L>0.02$. On the other hand, the sandwich plates have an total normalized thickness $\left(H_{\mathrm{c}}+2 h_{\mathrm{f}}\right) / L$ that is greater 0.1 , and stretching does not become dominant until $\delta_{\text {punch }} / L$ exceeds 0.15 . Plastic yielding of the sandwich plates occurs in the bending regime at $\delta_{\text {punch }} / L \cong 0.01$. At normalized deflections less than $\delta_{\text {punch }} / L \approx 0.1$, the punch force required to deform the sandwich plates is much higher than that for the solid plate. At very large deflections, the force required to deform the solid plate exceeds that for the sandwich plates since the entire plate material contributes to the stretch resistance. By contrast, the core of the truss core plate makes essentially no contribution to stretch resistance,

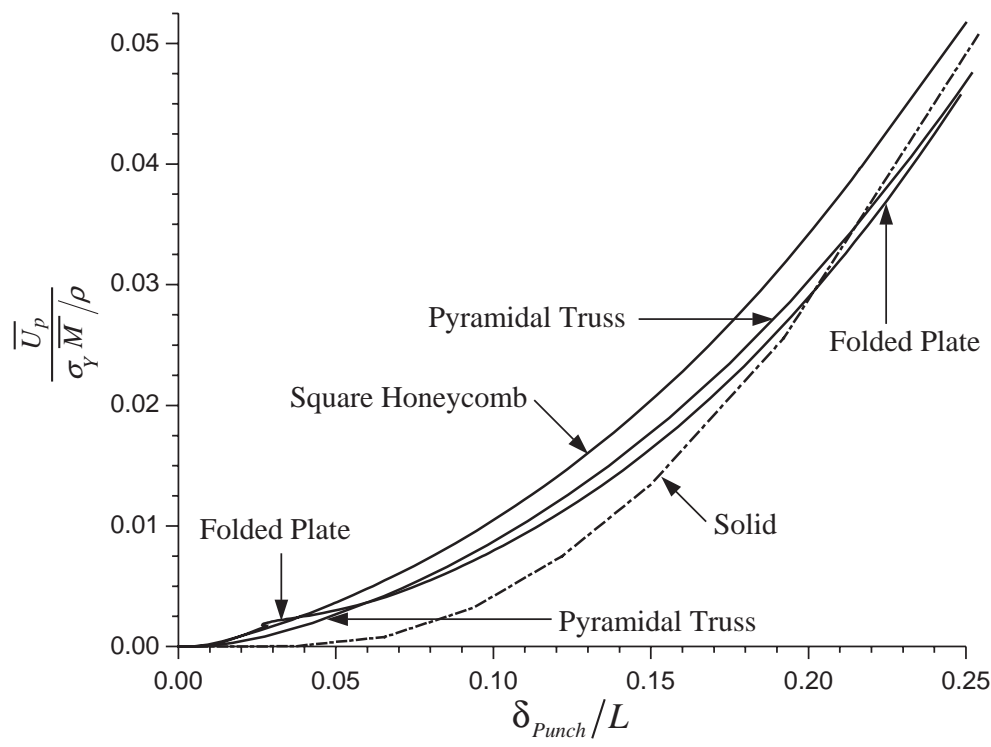

Fig. 7. Plastic energy dissipation for each of the four plates of Fig. 6. 
and it displays the lowest stretching strength of the four plates at large deflections. The folded plate core is aligned such that its webs contribute significantly to stretch, while only half of the webs of the square honeycomb core contribute to stretch.

For the force-deflection responses shown above, the plastic energy dissipated per unit area averaged over the plate, $\bar{U}_{\mathrm{p}}$, is presented as the dimensionless plot of $\bar{U}_{\mathrm{p}} /\left(\sigma_{\mathrm{Y}} \bar{M} / \rho\right)$ versus $\delta_{\text {punch }} / L$ in Fig. 7. For deflections satisfying $\delta_{\text {punch }} / L \leqslant 0.2$, all the sandwich plates absorb more energy than the solid plate of equal mass. The plate with the square honeycomb core absorbs the most energy at a give deflection, but its advantage over the other two sandwich plates in this respect is not great.

\section{Near-optimization of sandwich structures under blast load}

In this section, an effort to identify near-optimal configurations for each of the three sandwich plates subject to a moderately large blast will be made, and the results will be compared with the performance of the solid plate of equal mass. As discussed in Section 2, plates are sought that undergo the smallest deflection for specified mass, plate width, and blast impulse. Analogous optimization procedures for sandwich plates with foam metal cores have been described in [9]. To complement the optimization studies, plots of plastic dissipation will be shown, revealing essential aspects related to the blast performance of sandwich plates.

The results will be presented in a dimensionless form. The dimensionless mass per area of the plate is specified by $\bar{M} / \rho L$. As discussed in Section 1.2, the blast loading is imposed as an initial momentum impulse per unit area, $I$, in the form of a uniform velocity of the top face sheet of the sandwich plates or of the solid plate. The quantity $\sqrt{\sigma_{\mathrm{Y}} / \rho}$ has dimensions of velocity and $t\left(L / \sqrt{\sigma_{\mathrm{Y}} / \rho}\right)$ is a dimensionless time variable that scales the time-dependence of the plate response [1]. The dimensionless impulse is taken as $I /\left(\bar{M} \sqrt{\sigma_{\mathrm{Y}} / \rho}\right)$. For the sandwich plate with the pyramidal truss core in Section 2.1, the normalized maximum deflection defends on the other dimensionless variables according to

$$
\frac{\delta_{\max }}{L}=f\left[\frac{I}{\bar{M} \sqrt{\sigma_{\mathrm{Y}} / \rho}}, \frac{\bar{M}}{\rho L}, \bar{\rho}_{\mathrm{c}}, \frac{H_{\mathrm{c}}}{L}, \alpha\right] .
$$

Left implicit in this relation is the requirement that the stress-strain curve scales with $\sigma_{\mathrm{Y}}$, i.e., there is an unstated dependence on $\sigma_{\mathrm{Y}} / E$ and $N_{\varepsilon}$. All computations in this section are made using ABAQUS Explicit; the material is again characterized using Eq. (4) with $\sigma_{\mathrm{Y}} / E=0.002$ and $N_{\varepsilon}=0.17$. The importance of Eq. (11) is that for a given material it permits scaling with respect to plate width $L$, subject to constraints on the dimensional combinations. For example, since the results presented below are all restricted to $\bar{M} / \rho L=0.02$, scaling using Eq. (11) requires that both $H_{\mathrm{c}}$ and $\bar{M}$ scale with $L$. The general form (11) also holds for sandwich plates with folded plate cores, and it applies to square honeycomb cores if a dependence on $\alpha$ is replaced by a dependence on $B / H_{\mathrm{c}}$. For the solid plate, there is only a dependence on the first two dimensionless variables in Eq. (11). 

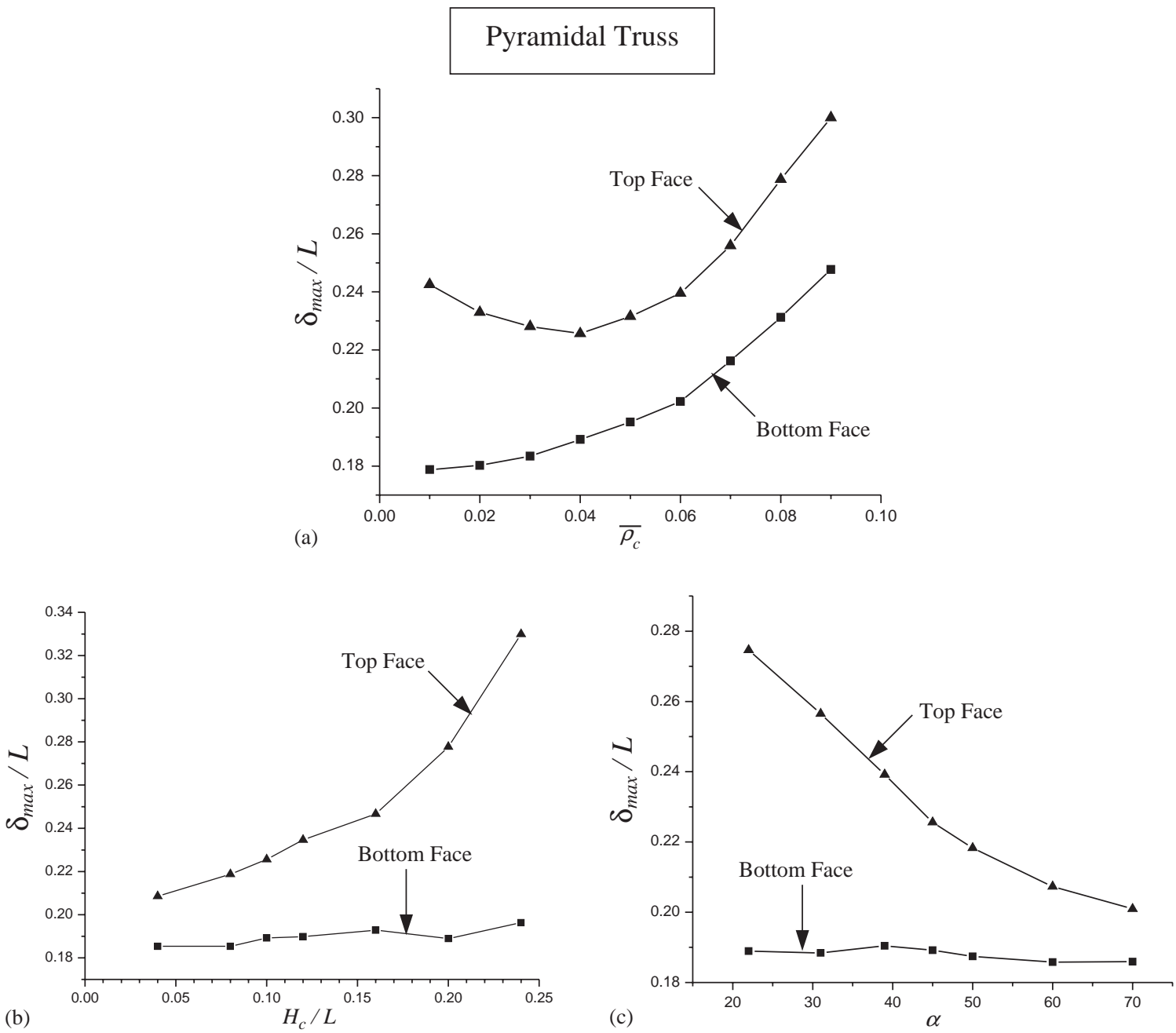

Fig. 8. Normalized maximum deflection $\delta_{\max } / L$ of the top and bottom face sheets of a sandwich plate with a pyramidal truss core with $\bar{M} /(\rho L)=0.02$ and subject to $I /\left(\bar{M} \sqrt{\sigma_{\mathrm{Y}} / \rho}\right)=0.25$ : (a) versus $\bar{\rho}_{\mathrm{c}}$ (with $H_{\mathrm{c}} / L=0.1$ and $\alpha=45^{\circ}$ ); (b) versus $H_{\mathrm{c}} / L$ (with $\bar{\rho}_{\mathrm{c}}=0.04$ and $\alpha=45^{\circ}$ ); and (c) versus $\alpha$ (with $\bar{\rho}_{\mathrm{c}}=0.04$ and $H_{\mathrm{c}} / L=0.1$ ).

\subsection{Searching for optimal configurations of the three types of sandwich structures}

Figs. 8a-c present the normalized maximum deflection $\delta_{\max } / L$ of the top and bottom face sheets of the sandwich plate with the pyramidal truss core as a function of the relative core density $\bar{\rho}_{\mathrm{c}}$, normalized core height $H_{\mathrm{c}} / L$, and angle of inclination $\alpha$, respectively. The nodes at which the deflections are evaluated are noted in Fig. 2b. The mass per unit area in these examples and in all the other cases presented in this paper is taken to be $\bar{M} / \rho L=0.02$. All points in these figures correspond to a relatively large impulse $\left(I /\left(\bar{M} \sqrt{\sigma_{\mathrm{Y}} / \rho}\right)=1 / 4\right)$ applied uniformly to the top face sheet. In Fig. 8a, the relative density $\bar{\rho}_{\mathrm{c}}$ of the plates is varied and with fixed values of normalized core height and angle $\left(H_{\mathrm{c}} / L=0.1, \alpha=45^{\circ}\right)$. As the relative density $\bar{\rho}_{\mathrm{c}}$ decreases from 0.09 to 
0.01 , the normalized maximum deflection $\delta_{\max } / L$ of bottom face sheet decreases monotonically, but the top face sheet experiences the smallest maximum deflection at a relative core density $\bar{\rho}_{\mathrm{c}} \approx 0.04$. Typically, the nominal compressive strain in the core, defined as compressive deformation per unit core height, is between $35 \%$ and $50 \%$ at the center of the plate, except for the two lowest core densities where the compaction strains exceed $50 \%$. The core trusses near the center of sandwich structure have fully buckled at this impulse level, but the truss core members near the edge of sandwich plate have not buckled because of constraint of the clamped edges.

In Fig. 8 b, the relative density is fixed at $\bar{\rho}_{\mathrm{c}}=0.04$, the angle of inclination is fixed at $\alpha=45^{\circ}$, and the normalized core height $H_{\mathrm{c}} / L$ is varied. In this case, the thickness of face sheets $h_{\mathrm{f}}$ obtained from Eq. (4) increases with decreasing the core height $H_{\mathrm{c}}$. The core height significantly affects the maximum deflection of top face sheet, but has relatively little influence on that of bottom face sheet. The effect of the angle of inclination $\alpha$, shown in Fig. 8c (with $\bar{\rho}_{\mathrm{c}}=0.04$ and $H_{\mathrm{c}} / L=0.1$ fixed), is similar. Truss members with large angles of inclination reduce the deflection of the top sheet but have little effect on the bottom face.

No further effort to optimize the pyramidal truss core plates has been made beyond the results shown in Fig. 8. Nevertheless, these results are sufficient to reveal that for such plates with $\bar{M} / \rho L=$ 0.02 , the deflection of the bottom face, $\delta_{\max } / L \cong 0.19$, is likely nearly the minimum possible for $I /\left(\bar{M} \sqrt{\sigma_{\mathrm{Y}} / \rho}\right)=1 / 4$. It is attained for a plate with $\bar{\rho}_{\mathrm{c}}=0.04, H_{\mathrm{c}} / L=0.1$, and $\alpha=45^{\circ}$.

A similar procedure has been executed for the sandwich plate with square honeycomb core, again for $\bar{M} / \rho L=0.02$ and $I /\left(\bar{M} \sqrt{\sigma_{\mathrm{Y}} / \rho}\right)=1 / 4$. The numerical results are shown in Fig. 9a-c. In Fig. $9 \mathrm{a}$, it is seen that both the bottom and top face sheets, experience the smallest maximum deflection, occurs at $\bar{\rho}_{\mathrm{c}}=0.04$ (with $H_{\mathrm{c}} / L=0.1$ and $B / L=0.1$ ). The nominal compressive strain in the core is less than $20 \%$ for almost all $\bar{\rho}_{\mathrm{c}}$, except for the lowest relative densities. Except possibly at very low relative densities, the honeycomb core sustains higher compaction loads than the pyramidal truss core, and thus undergoes less compaction. Variations of the normalized honeycomb size, $B / L$, (with $H_{\mathrm{c}} / L=0.1$ and $\bar{\rho}_{\mathrm{c}}=0.04$ ) in Fig. 9b shows that the minimum maximum deflection of both the top and bottom face sheets occurs for $B / L \cong 0.1$ or $B / H_{\mathrm{c}} \cong 1$. As $B / L$ increases, the top face sheet undergoes more and more localized bending between the honeycomb webs into the core cavities. This phenomenon contributes to the maximum deflection of the top face sheet shown in Fig. 9b. Fig. 9c shows that the minimum value of maximum deflection is obtained for a relatively thick core with $H_{\mathrm{c}} / L=0.22$ if $\bar{\rho}_{\mathrm{c}}=0.04$ and $B / L=0.1$. While the results of Fig. 9 do not constitute a full optimization for the square honeycomb core plate, it is clear that the minimum maximum deflection of the bottom face sheet will only be slightly less than $\delta_{\max } / L=0.125$ for plates with $\bar{M} / \rho L=0.02$ subject to $I /\left(\bar{M} \sqrt{\sigma_{\mathrm{Y}} / \rho}\right)=1 / 4$. Note that with $\bar{\rho}_{\mathrm{c}}=0.04$ and $H_{\mathrm{c}} / L=0.1$, precisely $20 \%$ of the total mass of the plate is in the core. For $H_{c} / L=0.22$, the fraction of mass in the core is $44 \%$.

The corresponding study for sandwich plates with folded plate cores is presented in Fig. 10 . With $H_{\mathrm{c}} / L=0.1$ and $\alpha=45^{\circ}$, the normalized maximum deflection has smallest value at $\bar{\rho}_{\mathrm{c}}=$ 0.05 and 0.04 for the top and bottom face sheets, respectively, as seen in Fig. 10a. The influence of the angle of inclination of the core webs, $\alpha$, with $H_{\mathrm{c}} / L=0.1$ and $\bar{\rho}_{\mathrm{c}}=0.04$ is shown in Fig. $10 \mathrm{~b}$. The maximum deflection of the top face sheet is fairly sensitive to $\alpha$, but the bottom face sheet displays little dependence on $\alpha$. Fig. 10c shows that, with $\bar{\rho}_{\mathrm{c}}=0.04$ and $\alpha=45^{\circ}$, the normalized maximum deflection of top face sheet increases monotonically with normalized core height $H_{\mathrm{c}} / L$, 

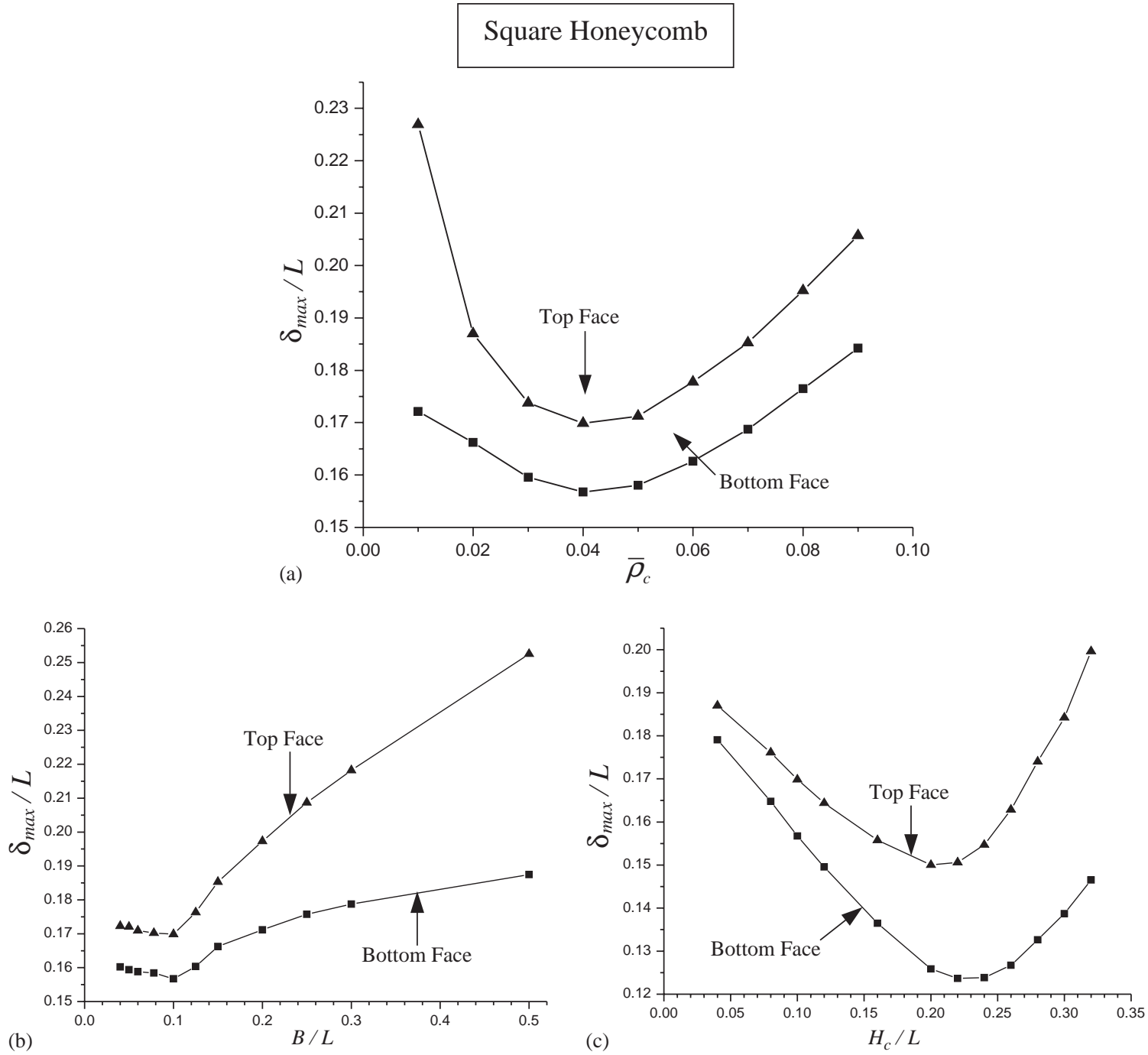

Fig. 9. Normalized maximum deflection $\delta_{\max } / L$ of the top and bottom face sheets of a sandwich plate with a square honeycomb core with $\bar{M} /(\rho L)=0.02$ and subject to $I /\left(\bar{M} \sqrt{\sigma_{\mathrm{Y}} / \rho}\right)=0.25$ : (a) versus $\bar{\rho}_{\mathrm{c}}$ (with $H_{\mathrm{c}} / L=0.1$ and $B / L=0.1$ ); (b) versus $B / L$ (with $\bar{\rho}_{\mathrm{c}}=0.04$ and $H_{\mathrm{c}} / L=0.1$ ); and (c) versus $H_{\mathrm{c}} / L$ (with $\bar{\rho}_{\mathrm{c}}=0.04$ and $B / L=0.1$ ).

while that of bottom face sheet has minimum deflection for $H_{\mathrm{c}} / L=0.16$. The minimum maximum deflection of the bottom face sheet of folded core sandwich plates is not expected to be much below $\delta_{\max } / L=0.14$ for plates with $\bar{M} / \rho L=0.02$ subject to $I /\left(\bar{M} \sqrt{\sigma_{\mathrm{Y}} / \rho}\right)=1 / 4$.

\subsection{Performance comparison for plates with $\bar{M} / \rho L=0.02$ subject to the same impulse}

Computations for maximum deflection for the three types of sandwich plates having normalized mass $\bar{M} / \rho L=0.02$ were carried out at difference levels of blast as measured by $I /\left(\bar{M} \sqrt{\sigma_{\mathrm{Y}} / \rho}\right)$ and presented in Fig. 11. The geometry of the plates with the truss and folded plate 

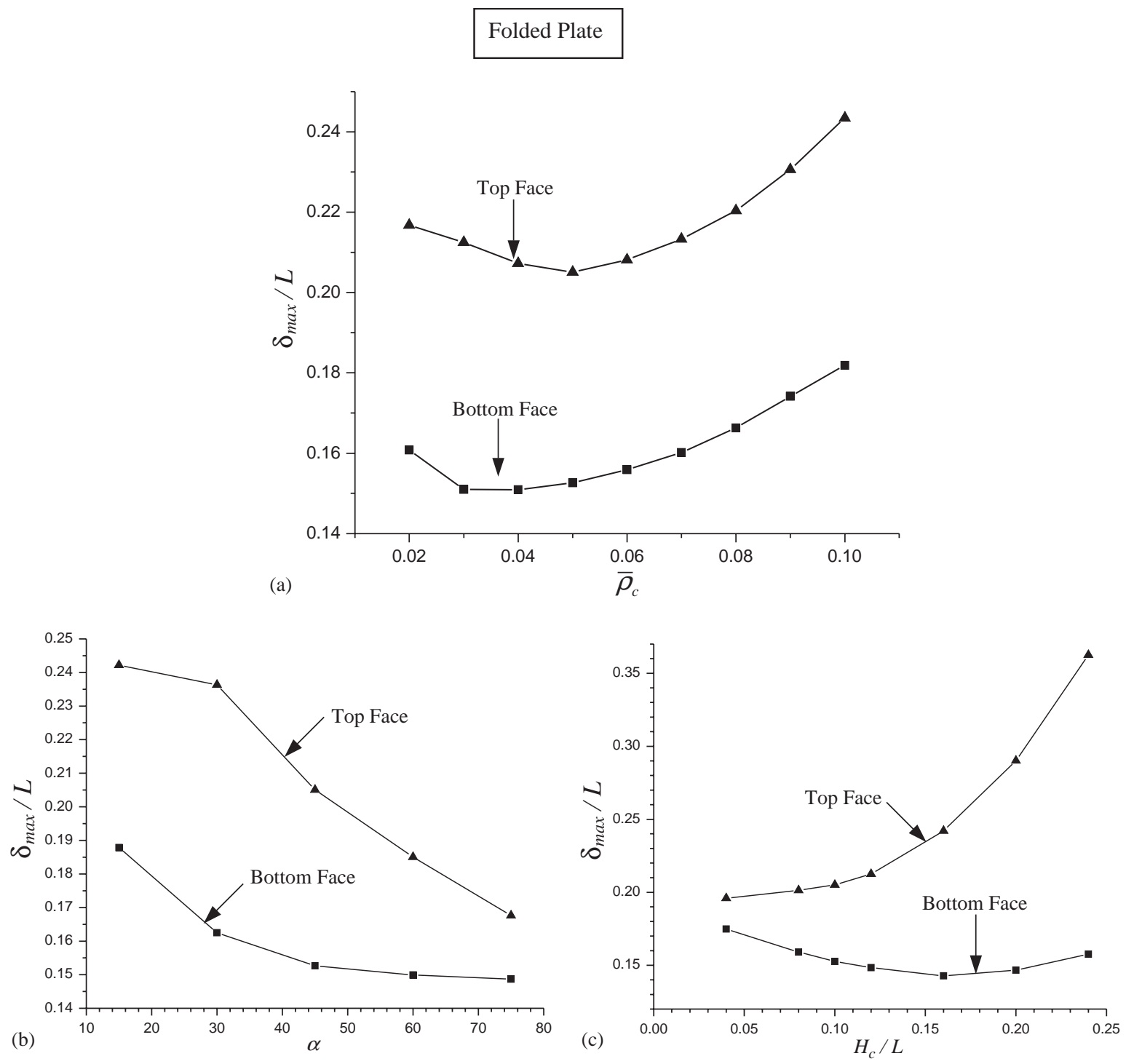

Fig. 10. Normalized maximum deflection $\delta_{\max } / L$ of the top and bottom face sheets of a sandwich plate with a folded plate core with $\bar{M} /(\rho L)=0.02$ and subject to $I /\left(\bar{M} \sqrt{\sigma_{\mathrm{Y}} / \rho}\right)=0.25$ : (a) versus $\bar{\rho}_{\mathrm{c}}$ (with $H_{\mathrm{c}} / L=0.1$ and $\alpha=45^{\circ}$ ); (b) versus $\alpha$ (with $\bar{\rho}_{\mathrm{c}}=0.04$ and $H_{\mathrm{c}} / L=0.1$ ); and (c) versus $H_{\mathrm{c}} / L$ (with $\bar{\rho}_{c}=0.04$ and $\alpha=45^{\circ}$ ).

cores are specified by $\bar{\rho}_{\mathrm{c}}=0.04, H_{\mathrm{c}} / L=0.1$, and $\alpha=45^{\circ}$, while the square honeycomb plate is specified by $\bar{\rho}_{\mathrm{c}}=0.04, H_{\mathrm{c}} / L=0.1$, and $B_{\mathrm{c}} / H_{\mathrm{c}}=1$. These parameters are fixed and not optimized at each specific impulse level. From the previous results for $I /\left(\bar{M} \sqrt{\sigma_{\mathrm{Y}} / \rho}\right)=1 / 4$, it is clear that the choice $H_{\mathrm{c}} / L=0.1$ has the most effect on the square honeycomb plates, leading to overestimates of the minimum maximum deflection by about $20 \%$. Results for the top face sheet are plotted in Fig. 11a and those for the bottom face in Fig. 11b. Included in each plot is the curve for a solid plate of equal mass. 

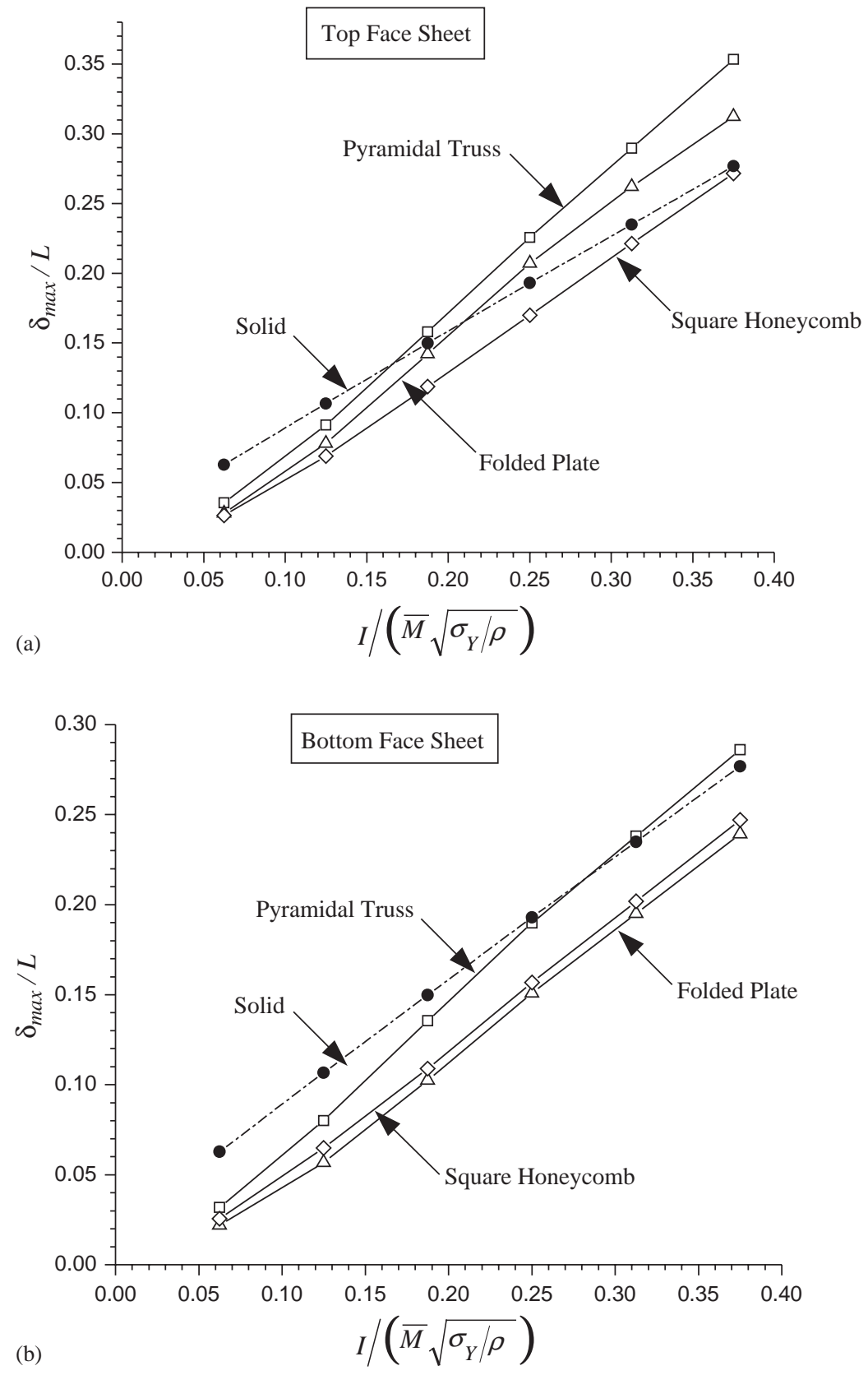

Fig. 11. The normalized maximum deflection $\delta_{\max } / L$ of partially optimized sandwich plates with the three core types subject to normalized momentum impulse $I /\left(\bar{M} \sqrt{\sigma_{\mathrm{Y}} / \rho}\right)$. The results for the solid plate of equal mass are also plotted for comparison. All plates have $\bar{M} /(\rho L)=0.02$. The sandwich plates have $\bar{\rho}_{\mathrm{c}}=0.04$ and $H_{\mathrm{c}} / L=0.1$ with $\alpha=45^{\circ}$ for the pyramidal truss and folded plate cores and $B / H_{\mathrm{c}}=1$ for the honeycomb core: (a) top face sheet and (b) bottom face sheet. 
Even if it could withstand the deformation without fracturing, no plate is likely to be allowed to sustain deflections much greater than $\delta_{\max } / L=0.2$ due to constraints on geometry change. Thus, for plates with $\bar{M} / \rho L=0.02$ the maximum momentum impulse that can be sustained is not likely be much larger than $I /\left(\bar{M} \sqrt{\sigma_{\mathrm{Y}} / \rho}\right)=0.3$ (cf. Fig. 11). For $I /\left(\bar{M} \sqrt{\sigma_{\mathrm{Y}} / \rho}\right)<0.3$, the square honeycomb plate and the folded core plate outperform the truss core plate and the solid plate in the sense that they sustain smaller deflections. The folded core plate has a slight advantage in bottom face deflection, while the square honeycomb core plate has slightly smaller top face deflection. In the range of smaller initial impulses, each of three sandwich plates outperforms the solid plate, and this advantage persists for blasts satisfying $I /\left(\bar{M} \sqrt{\sigma_{\mathrm{Y}} / \rho}\right)=0.25$ as measured by bottom face deflection. It must be emphasized that these comparisons are made at identical momentum impulse levels, which is appropriate for air blasts. The effect of fluid-structure interaction for water blasts has not taken account in this section. It will be accounted for in the comparison in the next Section 5.

\subsection{Plastic energy absorption of sandwich structures}

As emphasized in [1], there is a penalty associated with employing sandwich construction versus solid plate construction. Subject to the same initial momentum impulse, $I$, the ratio of initial kinetic energy per unit area imparted to the top sandwich face compared to that imparted to the solid plate is $h / h_{\mathrm{f}}$. The initial kinetic energy must be dissipated by the structure. Thus, if the goal for the sandwich plate is to sustain smaller deflections than the solid plate of equal mass when subject to the same initial impulse, then the sandwich plate must absorb more than twice the energy as its solid counterpart. The contributions of the component parts of the sandwich structures to energy dissipation are revealed by the time histories of plastic dissipation in the face sheets and core in Fig. 12a for pyramidal truss core plate and in Fig. 12b square honeycomb core plate. With $\bar{U}_{\mathrm{p}}$ as average plastic energy per unit area, the dimensionless contribution of each component of the plate, $\bar{U}_{\mathrm{p}} /\left(\sigma_{\mathrm{Y}} \bar{M} / \rho\right)$, is plotted as a function of dimensionless time, $t /\left(L / \sqrt{\sigma_{\mathrm{Y}} / \rho}\right) .{ }^{1}$ The specific cases are those discussed earlier for plates with $\bar{M} / \rho L=0.02$ subject to a large impulse, $I /\left(\bar{M} \sqrt{\sigma_{\mathrm{Y}} / \rho}\right)=1 / 4$, with $\bar{\rho}_{\mathrm{c}}=0.04, H_{\mathrm{c}} / L=0.1$ and $\alpha=45^{\circ}$ for the truss core plate and $\bar{\rho}_{\mathrm{c}}=0.04, H_{\mathrm{c}} / L=0.1$, and $B_{\mathrm{c}} / H_{\mathrm{c}}=1$ for the square honeycomb plate.

Each of the plots in Fig. 12 reveals that in the earliest stage of the deformation, when $t /\left(L / \sqrt{\sigma_{\mathrm{Y}} / \rho}\right)<0.05$, the core and the top face sheet undergo plastic deformation. In this stage, the face sheet flies into the core subjecting it to compression. By the end of this stage, the two face sheets are moving with nearly the same velocity. In the second stage there is very little further core compression and the entire composite plate undergoes bending and then in-plane stretching. For each of the two plates, almost all the plastic dissipation has occurred by $t /\left(L / \sqrt{\sigma_{\mathrm{Y}} / \rho}\right) \approx 0.8$. Subsequently, in the final stage, the plate undergoes elastic vibration, although this is not evident in plots of plastic dissipation. The total plastic energy dissipated attains about $90 \%$ of the initial kinetic energy. The initial kinetic energy is never fully dissipated plastically because of residual elastic stress (the main contribution) and continuing elastic vibratory motion. The main difference

\footnotetext{
${ }^{1}$ The normalization factor for the plastic dissipation per unit area, $\sigma_{\mathrm{Y}} \bar{M} / \rho$, reduces to $\sigma_{\mathrm{Y}} h$ for the solid plate. This is an appropriate choice because under a stretching strain $\varepsilon$, the plastic dissipation per area scales with $\sigma_{\mathrm{Y}} \varepsilon h$. The utility of the time scale, $L / \sqrt{\sigma_{\mathrm{Y}} / \rho}$, was demonstrated in [1] and is evident in Fig. 12.
} 

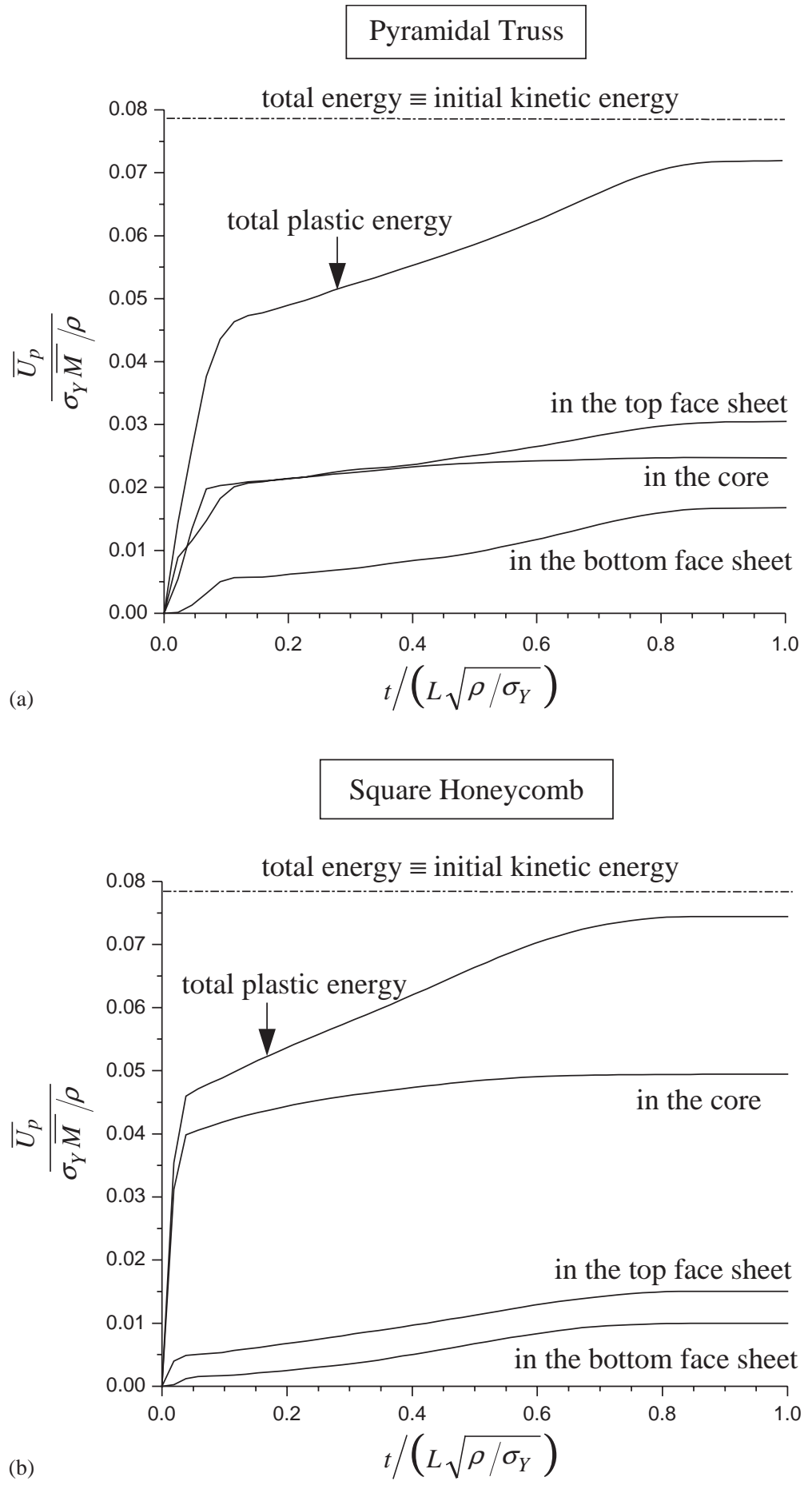

Fig. 12. The time history of plastic dissipation in sandwich plate with $\bar{M} /(\rho L)=0.02$ and subject to $I /\left(\bar{M} \sqrt{\sigma_{\mathrm{Y}} / \rho}\right)=0.25$ : (a) for pyramidal truss core with $\bar{\rho}_{\mathrm{c}}=0.04, H_{\mathrm{c}} / L=0.1$ and $\alpha=45^{\circ}$; and (b) for square honeycomb core with $\bar{\rho}_{\mathrm{c}}=0.04, H_{\mathrm{c}} / L=0.1$ and $B / H_{\mathrm{c}}=1$. 
in the responses of the two plate types is the substantially greater fraction of energy absorbed in the core of the honeycomb core plate. It should also be borne in mind that the honeycomb plate sustains a maximum deflection that is about $20 \%$ smaller than the truss core plate in this example.

Some sense can be had from the results of Fig. 12 of validity of the present approach whereby an initial momentum is imposed on the face sheet at $t=0$. To be valid, the approach requires that the pulse period, $t_{0}$, be small compared to the period of the first stage when the top face sheet flies into the core, i.e. small compared to $0.05 L / \sqrt{\sigma_{\mathrm{Y}} / \rho}$. For stainless steel plates with $L=1 \mathrm{~m}$, this requires that $t_{0}$ be small compared to $3.5 \times 10^{-4} \mathrm{~s}$. A blast pulse with $t_{0}=10^{-4} \mathrm{~s}$ meets this requirement, but only marginally so. Further studies investigating pulses applied for finite periods of time seem warranted, and these should account for fluid-structure interaction, as discussed next and in the Conclusions.

\section{The influence of fluid-structure interaction on the performance of sandwich plates in water blasts}

As remarked in Section 1.3, a plate in an air blast of sufficiently short duration will acquire nearly twice the free-field momentum impulse per area, $2 I_{0}$, unless the plate is exceptionally thin. By contrast, the time scale, $t_{*}$, in Eq. (2) for steel plates of thickness in the centimeter range subject to water blasts is comparable to typical blast periods, $t_{0}$. Consequently, the momentum impulse per area, $I$, imparted to the plate is much less than $2 I_{0}$. Moreover, as the example of Fig. 5 illustrates, less momentum is transferred to a plate that is thin (e.g. a face sheet of a sandwich plate) than to one that is thick (e.g. the equivalent mass solid plate). The ratio of the momentum acquired by the plate to the free-field momentum, $I / I_{0}$, is given by Eq. (1).

In what follows, we make use of $I / I_{0}$ to convert the results of Fig. 11 to a plot of $\delta_{\max } / L$ versus the free-field momentum, $I_{0}$, of a blast pulse in water. To make the conversion, the thickness of the solid plate and the face sheet of the sandwich plates must be specified. Thus, in converting the results of Fig. 11 for plates with $\bar{M} /(\rho L)=0.02$, steel plates are considered having half-width $L=1 \mathrm{~m}$ such that the thickness of the solid plate is $h=0.02 \mathrm{~m}$. Since all the sandwich plates have $\bar{\rho}_{\mathrm{c}}=0.04$ and $H_{\mathrm{c}} / L=0.1, h_{\mathrm{f}}=0.008 \mathrm{~m}$. With $t_{0}=1 \times 10^{-4} \mathrm{~s}$ and $c_{\mathrm{f}}=1500 \mathrm{~m} / \mathrm{s}$, $q=t_{0} / t_{*}=0.94$ for the solid plate, while $q=t_{0} / t_{*}=2.34$ for the face sheet of the sandwich plates. By Eq. (1), the factors accounting for the fluid-structure interaction are $I / I_{0}=0.76$ for the solid plate and $I / I_{0}=0.45$ for the face sheet. These factors have been used to obtain the results plotted in Fig. 13 from those in Fig. 11b for the maximum deflection of the bottom side of the plates.

The relative advantage of the sandwich plates over the solid plate of equal mass seen for air blasts in Fig. 11 is substantially enhanced for water blasts as seen in Fig. 13. For a maximum allowable deflection, a well-designed sandwich plate of $2-\mathrm{m}$ width can withstand a water blast almost three times that of a solid plate of equal mass. The plates with the folded plate core and the square honeycomb core are able to sustain higher blast impulses than the pyramidal truss core plate. In part, this is because these two cores contribute in-plane stretching resistance whereas the truss core does not, but the superior crushing strength of the honeycomb core is also a factor. 


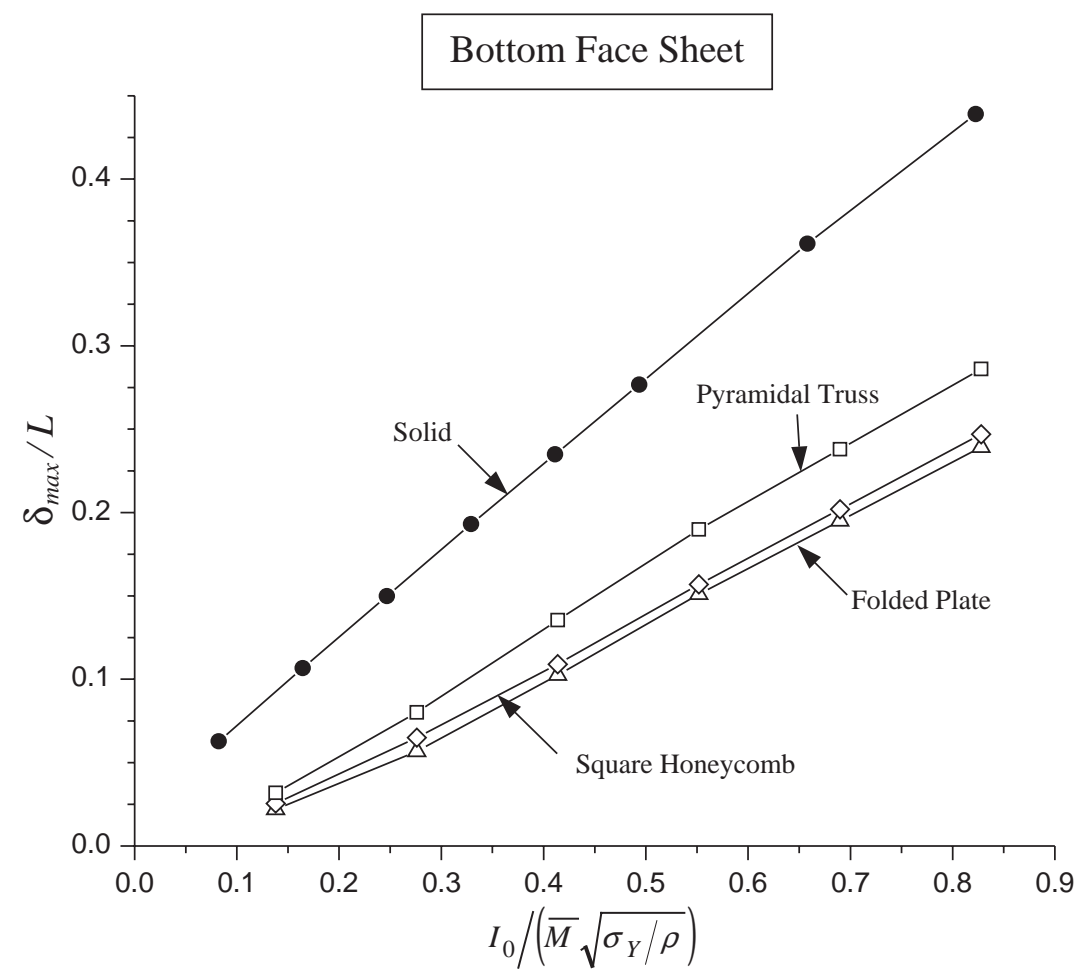

Fig. 13. Maximum deflection of the bottom side of the plates as a function of the normalized free-field momentum per unit area, $I_{0}$, for a blast pulse in water. All plates have $\bar{M} /(\rho L)=0.02$. The sandwich plates have $\bar{\rho}_{\mathrm{c}}=0.04$ and $H_{\mathrm{c}} / L=0.1$ with $\alpha=45^{\circ}$ for the pyramidal truss and folded plate cores and $B / H_{\mathrm{c}}=1$ for the honeycomb core. Further, these results are obtained from those in Fig. $11 \mathrm{~b}$ for the specific case $L=1 \mathrm{~m}$ with $h=0.02 \mathrm{~m}$ and $h=0.008 \mathrm{~m}$.

\section{Conclusions and suggestions for further research}

The examples studied in this paper indicate that there is considerable potential for exploiting metal sandwich plate construction for blast resistant structures. Square honeycomb and folded plate cores outperform truss cores, but all three types of sandwich plates are capable of sustaining larger blasts than the solid plate of equal mass. Complete optimizations have not been performed in this paper, but the range of geometry considered was sufficient to suggest that the configurations used to draw these conclusions were not far from optimal for design against a large blast. Fleck and Desphande [10] have developed analytic approximations of the behavior in the various stages of the sandwich plate response to the blast and have used these to conduct more extensive optimization studies.

While the present study indicates there are gains to be had from sandwich plate construction for air blasts, there is considerably more advantage in water blasts due to fluid-structure interaction. The present approach invokes two simplifications that will require more thorough examination in future work. First, the blast pulse period is assumed to be sufficiently short compared to the response time of the plate that the effect of the blast can be imposed as initial momentum, 
adjusted to account for the fluid-structure interaction. Second, this momentum is assigned to the face sheet of the sandwich plate towards the blast. The restraining effect of the rest of the plate structure is neglected in this assignment. Concomitantly, this approach assumes that the core and the back face sheet do not deform in the initial stage. Recent simulations that fully couple the fluid and the plate structure suggest that the present approach tends to overestimate the benefits of the fluid-structure interaction, especially for the sandwich plates [11]. Thus, the comparison between the water blast resistance of the sandwich plates and the solid plate of equal mass seen in Fig. 13 may be overly optimistic. This note of pessimism should be balanced against the fact that the results in Fig. 13 for the folded plate core and, especially, for the honeycomb core have not been fully optimized. In other words, super performance of the sandwich plates may be possible. Moreover, we have not considered the effect of allowing for differing thickness of the top and bottom face sheets, which can lead to even greater benefits for the sandwich plates.

In conclusion, in addition to the necessity of further work on identifying optimal configurations for specific applications, more work is required to assess effects of fluid-structure interaction and limits on deflection associated with various modes of fracture.

\section{Acknowledgements}

This work has been supported in part by the ONR under grants GG10376-114934 and N0001402-1-0700 and in part by the Division of Engineering and Applied Sciences, Harvard University.

\section{References}

[1] Xue Z, Hutchinson JW. Preliminary assessment of sandwich plates subject to blast loads. Int J Mech Sci 2003;45:687-705.

[2] Jones N. Structural impact. Cambridge: Cambridge University Press; 1989.

[3] Taylor GI. The pressure and impulse of submarine explosion waves on plates. In: Batchelor GK, editor. The scientific papers of Sir Geoffrey Ingram Taylor. vol. III: aerodynamics and the mechanics of projectiles and explosions. Cambridge: Cambridge University Press; 1963. p. 287-303.

[4] Cole RH. Underwater explosions. Princeton, NJ: Princeton University Press; 1948. p. 403-8.

[5] Swisdak MM. Explosion effects, properties: Part II—explosion effects in water. Report NSWC/WOL TR 76-116, Naval Surface Weapons Center, Dahlgren, VA, 1978.

[6] ABAQUS/Standard User's Manual. Version 6.0, Hibbit, Karlsson and Sorensen Inc., 2001.

[7] ABAQUS/Explicit User's Manual, Version 6.0, Hibbit, Karlsson and Sorensen Inc., 2001.

[8] Howard EB, Timothy LG, editors. Metals handbook desk edition. Cleveland, OH: American Society for Metals; 1985.

[9] Ashby MF, Evans AG, Fleck NA, Gibson LJ, Hutchinson JW, Wadley HNG. Metal foams: a design guide. London: Butterworth-Heinemann; 2000.

[10] Fleck NA, Deshpande VS. Blast resistance of clamped sandwich beams, Journal of Applied Mechanics, accepted for publication.

[11] Belytschko T, private communication. 